\title{
SPURIOUS ECCENTRICITIES OF DISTORTED BINARY COMPONENTS
}

\author{
Joel A. EATON \\ Center of Excellence in Information Systems, Tennessee State University, Nashville, TN 37209; eaton@donne.tsuniv.edu \\ Received 2007 April 26; accepted 2008 March 3
}

\begin{abstract}
I discuss the effect of physical distortion on the velocities of close binary components and how we may use the resulting distortion of velocity curves to constrain some properties of binary systems, such as inclination and mass ratio. Precise new velocities for $5 \mathrm{Cet}$ convincingly detect these distortions with their theoretically predicted phase dependence. We can even use such distortions of velocity curves to test Lucy's theory of convective gravity darkening. The observed distortions for TT Hya and 5 Cet require the contact components of those systems to be gravity darkened, probably somewhat more than predicted by Lucy's theory, but clearly not as much as expected for a radiative star. These results imply there is no credible evidence for eccentric orbits in binaries with contact components. I also present some speculative analyses of the observed properties of a binary encased in a nonrotating common envelope, if such an object could actually exist, and discuss how the limb darkening of some recently calculated model atmospheres for giant stars may bias my results for velocity-curve distortions, as well as other results from a wide range of analyses of binary stars.
\end{abstract}

Subject headings: binaries: spectroscopic — stars: oscillations — stars: individual (5 Cet, AX Mon, TT Hya)

Online material: machine-readable table

\section{INTRODUCTION}

Two recent papers suggest the time is ripe to look critically at the effect of stellar distortion on velocities of variable stars. In the first, Miller et al. (2007) found a slightly eccentric orbit for the Algol binary TT Hya, a system that has gone through mass exchange and, in so doing, surely circularized its orbit. In the second, Wood et al. (2004) used velocity variations calculated for a rotating prolate spheroid as a possible mechanism for explaining long-period velocity variations of AGB stars. The first paper assumes these distortions are irrelevant; the second, that they are crucial.

Solutions of the velocity curves of close binary systems often have small eccentricities thought to be spurious. Lucy \& Sweeney $(1971,1973)$ argued that most of these were statistical flukes resulting from errors of measurement. However, as we shall see in $\S 3$, there are circumstances in which such eccentricities result from interesting physical processes, and for which the resulting distortions may be used to extract useful information about the mass ratios and inclinations of these systems. Such distortions from proximity effects were actually predicted by Sterne (1941), explored by Wilson \& Sofia (1976) as a way of determining mass ratios and inclinations of $\mathrm{X}$-ray binaries, and discussed in passing by Wilson (1979), but otherwise they do seem to have been ignored as a way of extracting information about binaries.

Red giants often have unexplained secondary periods much longer than their radial fundamentals (e.g. Wood et al. 2004). They manifest uncomfortably large radial-velocity variations on those periods (Wood et al. 2004; Hinkle et al. 2002), which imply pulsational excursions of the order of $30 \%$ of the radius of the star. These variations remain one of the burning mysteries of stellar astronomy (Derekas et al. 2006). They seem to have the sort of color variations expected of radial pulsation, yet there is no known mechanism for giving such long periods.

I shall explore the implications of distortion on observed radial velocities, starting with an assessment of the rotating spheroids of Wood et al. in $\S 2$, then exploring how a binary encased in a common envelope might appear to us in $\S 2.1$, and finally dis- cussing the much less speculative effects of distortion on radial velocities of close binary components in $\S 3$.

\section{ROTATION OF A PROLATE SPHEROID}

Wood et al. (2004) used a prolate spheriod rotating about its short axis to model velocity variations of AGB stars, although they ultimately rejected that idea. Since such a figure will project symmetrically onto the sky about its rotation axis, there would be no net velocity variation for a uniformly illuminated disc. So these variations must occur solely from the effects of limb and gravity darkening over the distorted surface (P. Wood 2007, private communication; Sterne 1941).

Let us make some standard assumptions about the rotating spheriod. Give it axes $a$ and $b$ in its equatorial plane and, for simplicity, $b$ toward its rotational pole. Assume the mass is concentrated to its center, so the local gravity is proportional to $1 / r^{2}$, and let the gravity darkening be convective, $F_{\text {bol }} \sim \nabla \Omega^{g}$, with $g=0.32$ following Lucy (1967), where $\Omega$ is the gravitational potential. Assume total darkening to the limb $(x=1.0)$, a safe assumption for such cool stars. Finally, assume that the radial velocity of the centroid of light is the radial velocity measured for the star. Now, if we write a computer program for these somewhat questionable assumptions, we can calculate the light and velocity curves for such a star, if any such object were to exist.

Figure 1 shows some velocity curves for a prolate spheriod with $a=0.50$ and $b=0.25$, a rather extreme case that illustrates the model pretty well. For this combination of parameters, we get light variation of about $1.9 \mathrm{mag}$ at $i=90^{\circ}$, somewhat larger than seen in the AGB stars. The solid and dashed curves have $x=1.0$ with $g=0.0$ and $x=0.0$ with $g=0.32$, respectively, to show the separate effects of limb and gravity darkening, which seem to cancel each other out in this calculation. The dotted curve, for $x=1.0$ with $g=0.32$, shows the combined effect. Both limb and gravity darkening obviously cause significant velocity variations mimicking an eccentric orbit. The curve for pure limb darkening in Figure 1 may be fit with an orbit in which 


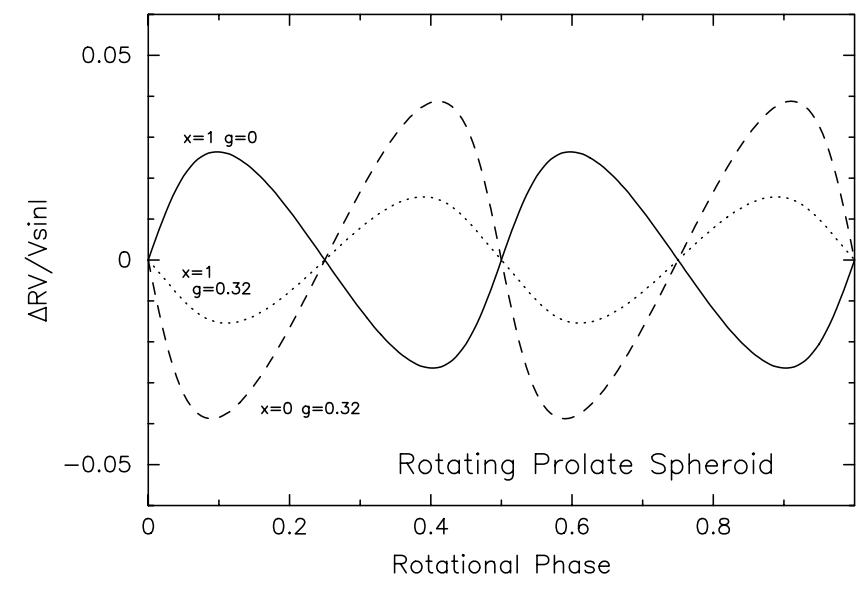

FIG. 1.- Velocity curves of a prolate spheroid with a $2: 1$ aspect ratio. The three curves show the effects of limb darkening alone (solid), of gravity darkening alone (dashed), and the combination of limb and gravity darkening (dotted). All assume $i=90^{\circ}$.

$e=0.21$ and $\omega=270^{\circ}$. The case of pure gravity darkening gives $e=0.22$ and $\omega=87^{\circ}$. Other aspect ratios $(b / a)$ give similar results but smaller amplitudes and somewhat smaller eccentricities for less elongation. More elongation gives very slightly bigger eccentricities.

These calculations for a rotating prolate spheroid do not represent the sort of long-period velocity variation observed in AGB stars. The eccentricities we find for this ellipsoidal model $(e \sim 0.2)$ are significantly smaller than the formal eccentricities of cool giants when compared to either the largest values measured or typical ones. Fitting the velocity shifts observed for nine stars with elliptical orbits gave $e$ in the range $0.08-0.49$ and $\omega$ in the range $195^{\circ}-418^{\circ}$ (Hinkle et al. 2002; Wood et al. 2004), with medians $e=0.33, \omega=251^{\circ}$, and $K=2.3 \mathrm{~km} \mathrm{~s}^{-1}$. The phase dependence of the calculated theoretical effect for gravity darkening is wrong in that it gives a rapid drop in velocity, as in a classical radial pulsation, not in the observed rapid rise. Furthermore, the velocity variation in these calculations $(\Delta \mathrm{RV} / v \sin i \sim 0.04)$ is small enough to require a rotational velocity $\left(58 \mathrm{~km} \mathrm{~s}^{-1}\right.$ for $K=2.3 \mathrm{~km} \mathrm{~s}^{-1}$ ) prohibitively large for AGB stars, for which $v_{\text {rot }} \lesssim 3 \mathrm{~km} \mathrm{~s}^{-1}$ (Olivier \& Wood 2003).

\subsection{Speculations about an Encased Binary}

It is not clear that any star, especially an AGB star, would be a prolate spheroid rotating about its fixed center of mass. The only way I can see to get a significant elongation is through tidal distortion in a close binary system, and the effects of that distortion on the velocities can be rather subtle, as we shall see in $\S 3$. Wood et al. argue that some of these AGB stars may be coalesced binaries, presumably with double cores encased in a common envelope to account for the prolate shape, a radically imaginative idea deserving a closer look.

The size of such an object presents another fundamental problem with trying to use a rotating prolate spheroid to explain the light and radial-velocity variations of AGB stars. If the star has a double core, its period and linear scale are fixed by Kepler's laws. Consider, for example, a star with a double core having a period of 1588 days (4.35 yr), as discussed by Wood et al. for $\mathrm{Z}$ Eri. It would have a semimajor axis of $3 \mathrm{AU}\left(640 R_{\odot}\right)$ for two $0.75 M_{\odot}$ components. Even in a binary consisting of two $1.0 M_{\odot}$ components with a period of 750 days, the semimajor axis would be about $440 R_{\odot}$. The radii of these objects would be of the order of their semimajor axes. Wood et al. estimate radii near $170 R_{\odot}$ for the stars with long secondary periods, so encased binaries with the right periods to explain the observed long-term velocity variations would seem to be too large. Also, if these stars were synchronously rotating, admittedly a completely unphysical condition, our two examples would have $v_{\text {rot }}$ of 20 and $30 \mathrm{~km} \mathrm{~s}^{-1}$, respectively, much larger than the $v \sin i$ 's of AGB stars.

The idea of a binary totally encased in a common envelope seems preposterous, since the gravitational equipotential surfaces above the two cores, which presumably help control the density structure of the star, become very complicated with three Lagrangian points at which the gravitational acceleration vanishes (e.g., Kopal 1959, their Figs. 7-7 through 7-10). However, this may be no worse than the generally accepted idea of a contact binary containing a single such Lagrangian point. Given these circumstances, however, it is not clear to me that any star could be stable with a surface bigger than in a typical contact binary (one with its surface between the first and second Lagrangian surfaces).

A more fundamental problem with this idea is the question of solid-body rotation. For a synchronous binary bigger than its second Lagrangian surface, the centrifugal potential dominates, and the surfaces of constant potential (normally the level surfaces) are unbound. Any star containing a double core must, therefore, be rotating slower than synchronously in its outer layers. I shall leave it to others to estimate the time the churning going on inside such an object would take to dissipate the angular momentum of the encased core. However, in this case, second-order effects of rotation cannot dominate the light or radial velocity of the star. Instead it must be subject to a nonradial pulsation driven by the encased binary. In this regard it is no different from other nonsynchronous binaries, such as the components of eccentric systems discussed by Wilson (1979).

Now, in the remote possibility that binaries with an encased core can actually exist long enough for us to observe them, just what might they look like? We can make some simplifying assumptions that let us calculate light and velocity curves for such an object. As a first stab, I have considered a system that is not rotating at all. This eliminates the centrifugal potential and makes the gravitational potential simply

$$
\Omega=1 / r_{1}+q / r_{2}
$$

where $r_{1}$ and $r_{2}$ are the distances of some point from the two components of the binary, the origin of coordinates is the binary's center of mass, and $q=M_{2} / M_{1}$ is the mass ratio of the system. The surface corresponding to an equipotential of this equation is shaped roughly like a prolate spheroid for equal masses $(q=1.0)$ and a large enough radius. For other mass ratios, the shape is more distorted and becomes rather biological at smaller radii. We can calculate the light and velocity curves for such objects if we make an assumption about how surface brightness changes over the star. I have made the simplifying assumption that the surface is always given by an equipotential, that this figure rotates with the binary system (i.e., has no phase lag), and that the surface brightness is determined by limb and gravity darkening as in a normal synchronously rotating binary. Actually, the surface brightness would be determined by the driven pulsation, so these assumptions are clearly bogus. However, they do give us a way to estimate the effect of having an encased binary as a star's core, at least to first order.

For these assumptions we may calculate the pulsational velocities, assumed radial, by comparing points equal distances apart in azimuth and dividing by the time it takes the binary to rotate that amount. Since in the calculations, we divide the star's 


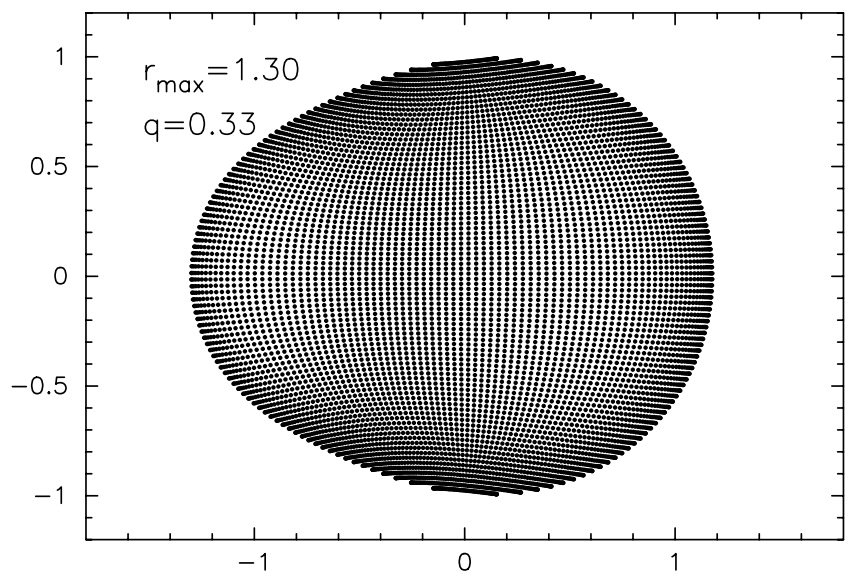

FIG. 2.-Distribution of surface elements in a representative calculation for an encased binary system at phase 0.25 , where the two components would have their maximum separation.

surface into a number of sectors of azimuth, each $\Delta \lambda$ wide in radians, the local pulsational velocity along the star's radius is

$$
V_{j}=\frac{d \lambda}{d t} \frac{d r}{d \lambda}=\frac{2 \pi}{P} \frac{a\left(r_{j+1}-r_{j-1}\right)}{2 \Delta \lambda} i,
$$

where $a$ and $P$ are the semimajor axis and period of the encased binary, $r_{j}$ is the radius in units of $a$, and index $j$ represents the steps in azimuth. The spheriodal pattern, rotating through the surface of the star, breaks the surface up into four sectors with alternating positive and negative pulsational velocities. These, in turn, project into our line of sight in a way that gives the star a roughly sinusoidal velocity variation on half the period of the encased binary.

Given the foregoing assumptions, we may calculate the properties of the encased binary to first order. Figure 2 shows roughly what such a star with a mass ratio of 0.33 and a radius of 1.3 times the semimajor axis might look like. Figure 3 shows some light curves for this sort of object, as well as for the two limiting contact binaries. Figure 4 shows some velocity curves. The important thing to note is that, for equal masses, the object gives a light

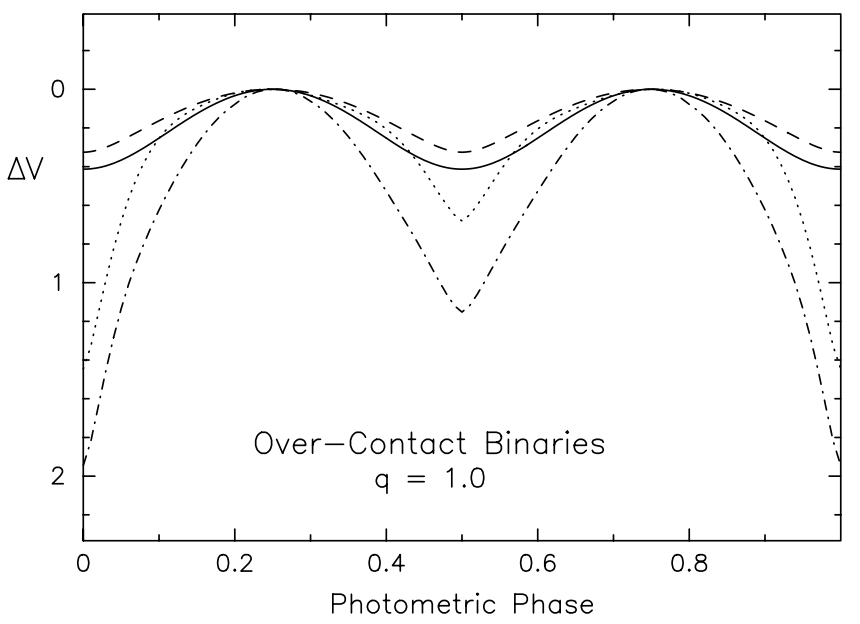

FIG. 3.- Light curves for an encased binary and two limiting contact binaries. The curves are as follows: solid, nonrotating encased binary with $r_{\max }=1.3 a$ and the expected limb and gravity darkening; dashed, encased binary without any limb or gravity darkening; dotted, contact binary with both stars just overfilling their Roche lobes (first Lagrangian surface); dot-dashed, contact binary filling its outer contact (second Lagrangian) surface. All these calculations assume $i=90^{\circ}$.

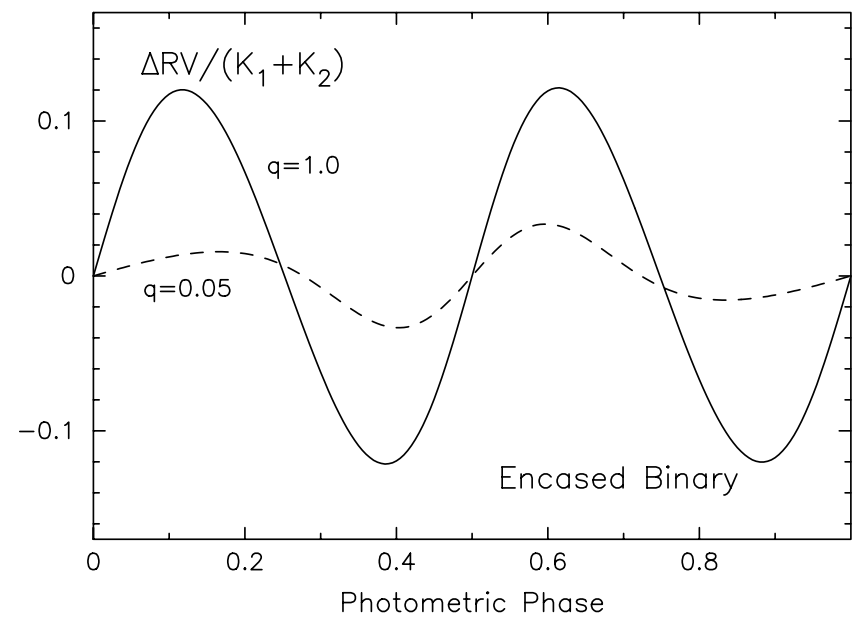

FIG. 4.- Velocity curves calculated for two encased binaries with $R=1.3 a$.

variation on half the orbital period of the encased binary. The radial-velocity variation is also on this period. For different mass ratios, the degeneracy in the light variation goes away, but the radial velocities still vary on half the orbital period in a peculiar way. If we ever were to find a star in the common-envelope stage, it would most likely have the light variations of a contact binary (e.g., dotted and dot-dashed curves in Fig. 3), but with velocity variations on half the photometric period.

\section{SPURIOUS ECCENTRICITIES FROM TIDAL DISTORTION}

The contact components of Algol binaries really are like prolate spheriods (actually, triaxial ellipsoids), but only to first order (e.g., Russell 1912). Instead, they have a complicated asymmetric figure if they actually follow the contours of their Roche lobes. These lobes are symmetric about a line between the two component stars, so the distortions would be symmetric about conjunctions. However, they would be different for the two conjunctions in a way that distorts the velocity curve. We can predict this distortion with binary modeling software and test it with precisely measured velocities of contact components of close binaries (Wilson \& Sofia 1976).

The new date we are using here are radial velocities from the Tennessee State University (TSU) Automatic Spectroscopic Telescope (AST; Eaton \& Williamson 2007). These echelle spectra of roughly 30,000 resolution cover the wavelength range 6000-7100 A. We reduced and analyzed them with standard pipeline techniques to derive the radial velocities for 5 Cet (HD 352) and AX Mon (HD 45910), given in Table 1 (available in full on

TABLE 1

Spectroscopic Data

\begin{tabular}{|c|c|c|}
\hline $\begin{array}{c}\text { HJD } \\
(2,400,000+)\end{array}$ & $\begin{array}{c}\mathrm{RV} \\
\left(\mathrm{km} \mathrm{s}^{-1}\right)\end{array}$ & Star \\
\hline $52897.9971 \ldots \ldots \ldots \ldots \ldots \ldots \ldots \ldots \ldots$ & -8.25 & HD 352 \\
\hline $52900.9680 .$. & -3.95 & HD 352 \\
\hline 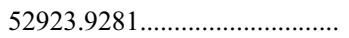 & 22.48 & HD 352 \\
\hline $52959.6797 \ldots \ldots$ & -14.17 & HD 352 \\
\hline $52970.7973 \ldots \ldots$ & -23.54 & HD 352 \\
\hline 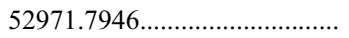 & -23.58 & HD 352 \\
\hline
\end{tabular}

Note.-Table 1 is published in its entirety in the electronic edition of the Astrophysical Journal. A portion is shown here for guidance regarding its form and content. 


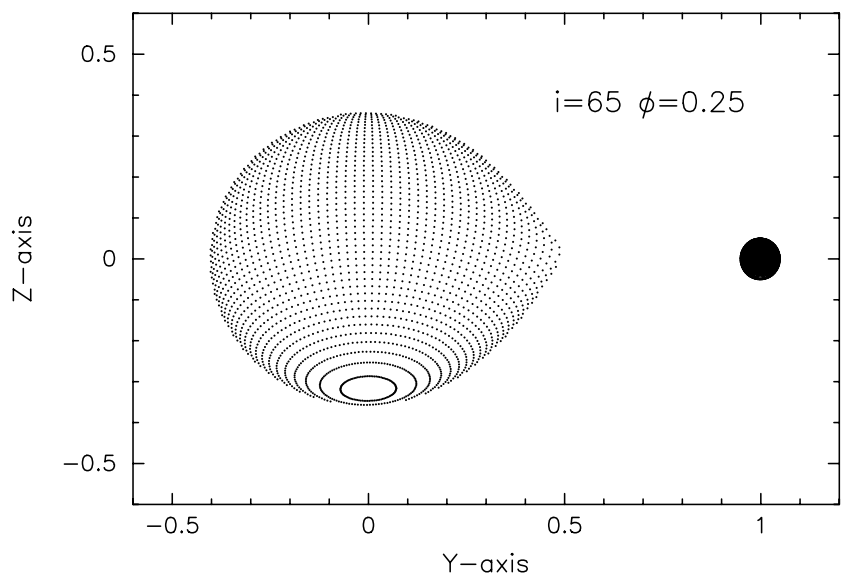

FIG. 5.-Distribution of surface elements in a typical calculation for a semidetached system at phase 0.25 when the bigger, distorted star is approaching. The two stars obviously have equal masses in this calculation.

line). Velocities measured for bright stars such as 5 Cet have an external precision of $\sim 0.11 \mathrm{~km} \mathrm{~s}^{-1}$ RMS (Eaton \& Williamson 2007). Listed in the table (see printed excerpt) are (1) HJD, the Heliocentric Julian Date of observation (minus 2,400,000), (2) RV, the radial velocity of the cool star, and (3) a tag identifying the star by its HD number.

\subsection{Modeling the Velocity Distortions}

We can calculate the effect of tidal distortion combined with limb and gravity darkening on the velocities of Algol components if we make an assumption about how the measured velocity is related to surface brightness on the star. I shall make the simplifying assumption ${ }^{1}$ that a line profile is constant over the disc, and that the observed velocity is just the local velocity weighted by intensity and averaged over the visible disc. For a sphere, the velocity calculated in this scheme is just the velocity at disc center, so there are no deviations from the velocity of the center of mass. For a distorted binary component, we can calculate the deviation by averaging $y^{*} \Delta A^{*} I_{\lambda}$ over the component, where $y$ is the distance perpendicular to the rotation axis pro-

\footnotetext{
1 One should keep in mind that there may be small, third-order systematic errors introduced by this approach (Winn et al. 2005).
}

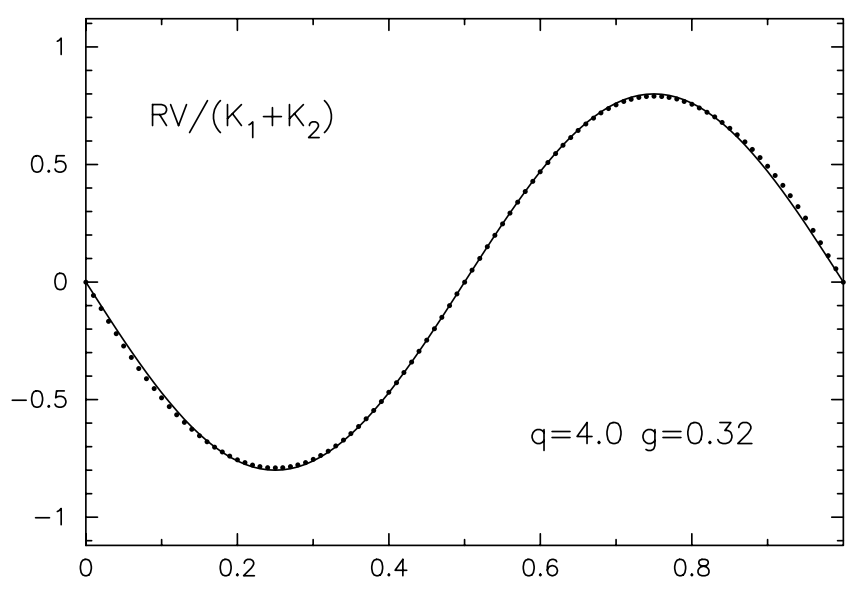

jected into the sky, $\Delta A$ is the projected area of a surface element in the calculation, and $I_{\lambda}$ is the intensity as determined by limb and gravity darkening. We can get this information from computer programs for calculating light and velocity curves of binaries, such as Wilson's (1979), and I have coded it into my own program (e.g., Eaton et al. 1993), which is easier for me to understand.

Figure 5 shows the distribution of surface elements in a typical calculation for phase 0.25 when the bigger, distorted star is approaching. The second component (generally hotter and smaller) is to the right (at $y=1.0$ ) in this coordinate system. At the phase illustrated, the left side of the star (negative $y$ 's) contributes more light than the right side, in spite of there being more area to the right, because of limb and gravity darkening. Some results are given in Figure 6, where we see the effect of the distortion on a typical velocity curve at the left and some calculated deviations in the right panel. I have expressed these curves in terms of the total velocity amplitude of the system $\left(K_{1}+K_{2}\right)$, since the calculated quantity $\left(\sum y^{*} \Delta A^{*} I_{\lambda}\right)$ is the displacement of the centroid of light from the rotation axis in units of the separation of the two stars. Three things are clear in Figures 6 and 5. First, the effect on the velocity curve is subtle but systematic. Second, the magnitude of the effect would depend upon mass ratio mostly through changes in the relative velocity amplitudes of the two stars: the less massive the undistorted companion, the smaller the effect on the distorted star's velocity curve. Third, the effect is smaller at lower inclination because the roughly symmetric gravity-brightened pole contributes more of the light.

Figure 6 shows how the mass ratio affects distortion of the velocity curve. Figures 7 and 8 show the effects of inclination and gravity darkening. Both are significant. By contrast, the effect of limb darkening is more moderate. ${ }^{2}$ The large dependence on inclination in Figure 7 means we can use these velocity-curve distortions to estimate inclinations of noneclipsing Algol binaries. I have plotted these curves for binaries against photometric phase, for which the zero point is superior conjunction of the distorted star, the phase of minimum light in ellipsoidal variation (e.g., Hall 1990).

\footnotetext{
2 If we adopt linear limb darkening, as I have, a change of $\Delta x_{V}=0.1$ corresponds to $\Delta g \sim-0.012$ or $\Delta i \sim 0.8$. The effect on mass ratio would be rather large, however (see Fig. 6).
}

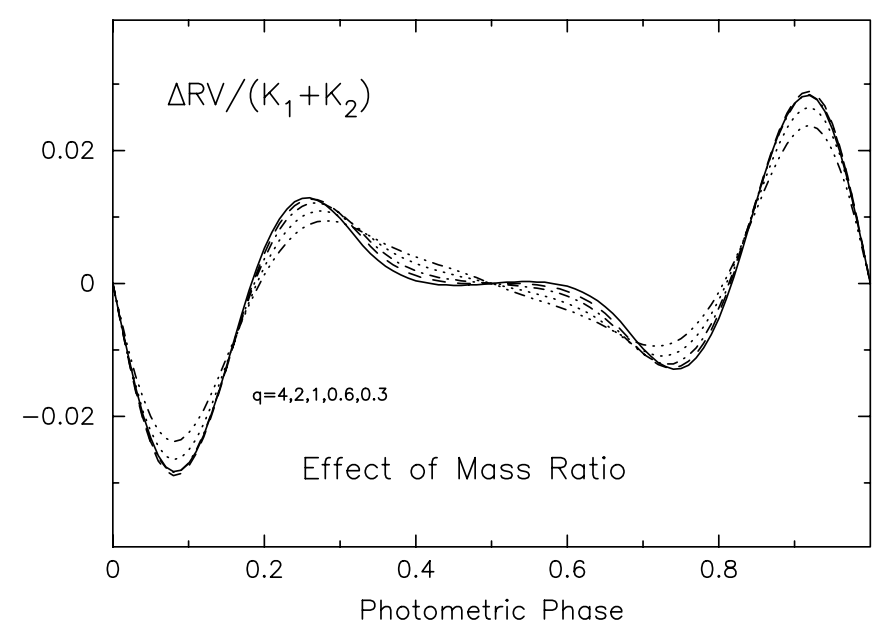

FIG. 6.-Effect of the distortion on a typical velocity curve (left), and some calculated deviations (right). The top panel shows a calculation for $i=85^{\circ}$ and $q=$

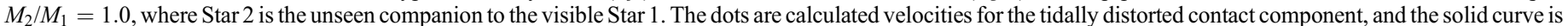

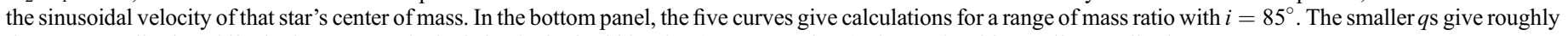
the same amplitude, while the larger $q$ s typical of classical Algol binaries $(q=2.0$ and 4.0$)$ give noticeably smaller amplitudes. 


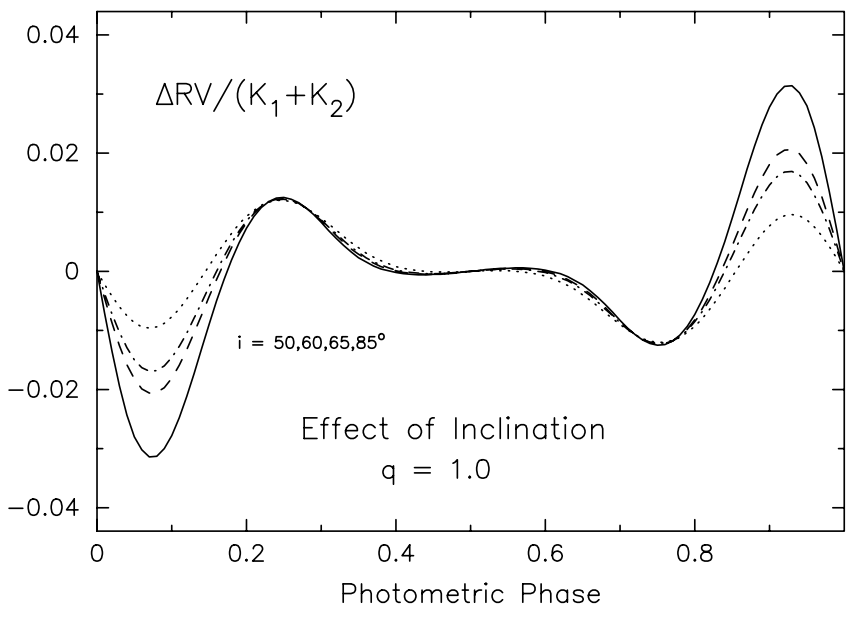

FIG. 7.-Effect of orbital inclination on the distortion of the velocity curve. Inclination ranges from $50^{\circ}$ (dotted curve) to $85^{\circ}$ (solid curve). Note how different this dependence on inclination is for the two conjunctions.

\subsection{Tests for Some Actual Stars}

HD $352=5$ Cet (also, perversely, AP Psc) is a close semidetached binary system with a giant contact component (e.g., Eaton \& Barden 1988). It is rather bright and single-lined. We have obtained 126 observations of it over a period of 3 years with the AST. The velocity curve deviates from the sinusoidal shape expected for a spherical star in a circular orbit by a noticeable amount (see Fig. 9). We can reduce the obvious systematic errors of the fit by allowing an eccentricity, $e=0.033 \pm 0.002$ with $\omega=88.3 \pm 0.1$. However, there are still systematic deviations from the fitted curve. Figure 10 shows the deviations from a sinusoid. They are obviously of the same shape, and if we apply the right values for the physical parameters of the system (see $\S 3.3$ ), of roughly the right amplitude as the theoretical calculations in Figure 6. You may note that the data scatter by roughly the expected external error $\left(0.1 \mathrm{~km} \mathrm{~s}^{-1}\right)$ about this relation.

The spurious eccentricity we have found for 5 Cet has essentially the same magnitude and orbital orientation as Miller et al. (2007) claimed for TT Hya. Furthermore, the velocity curves of the cool components of all Algol binaries should show spurious

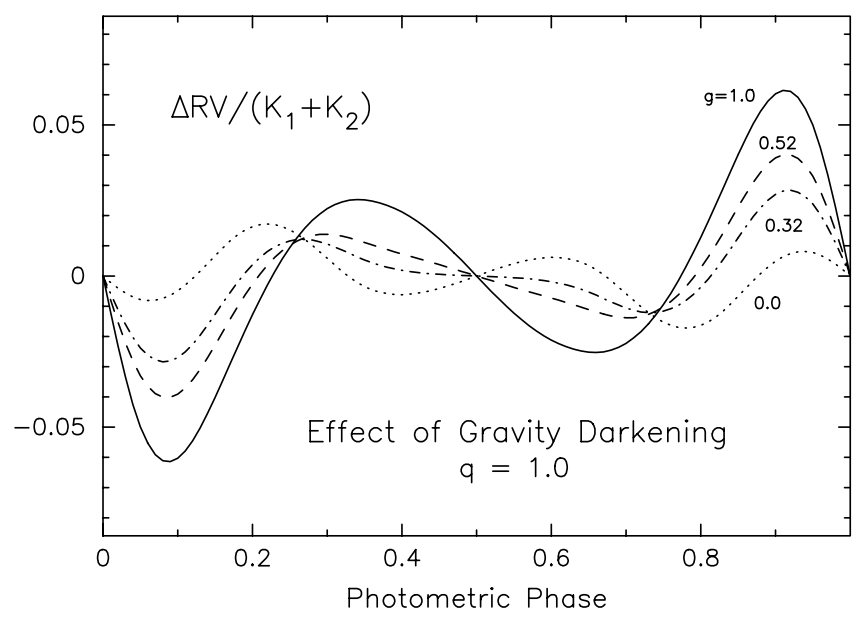

FIG. 8.-Effect of gravity darkening on the distortion of the velocity curve. Curves are as follows: dotted for $g=0.0$ (no gravity darkening), dot-dashed for $g=0.32$ (Lucy's theoretical value), dashed for $g=0.52$, and solid for $g=1.0$ (radiative-von Zeipel-gravity darkening).

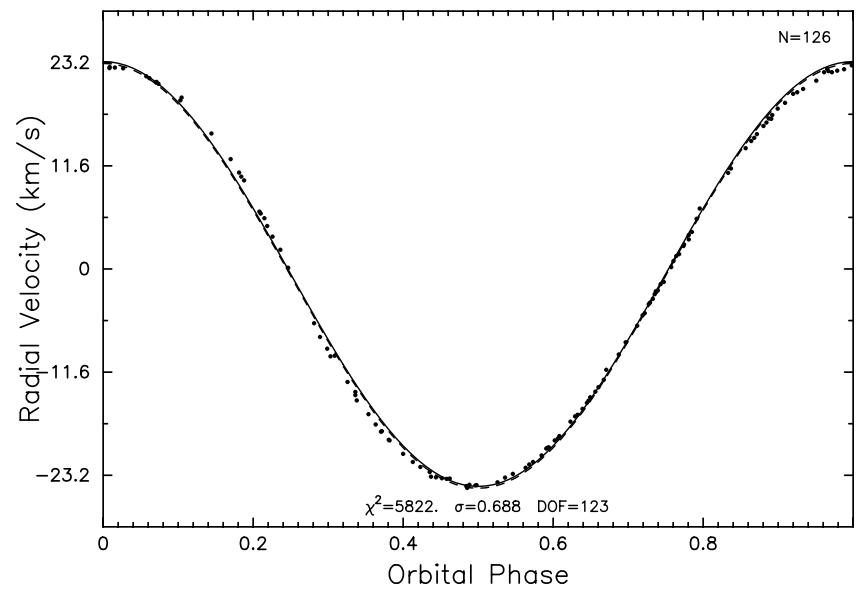

FIG. 9.- Fit of a circular orbit to measured velocities for 5 Cet. The RMS deviation for this fit is $\sigma=0.69 \mathrm{~km} \mathrm{~s}^{-1}$; the systematic deviation is obvious. Compare this graph to the left panel of Fig. 6 .

eccentricities of this type $\left(e \sim 0.02-0.04, \omega \sim 90^{\circ}\right)$. That they do not simply means that the measured velocities have not been precise enough to detect this effect, as Lucy \& Sweeney told us. However, we may test this idea further with 177 radial velocities from the AST for AX Mon (HD 45910), for which we have a preliminary solution. In that case both circular and elliptical orbits fit the data to $\sigma \approx 0.9 \mathrm{~km} \mathrm{~s}^{-1}$. The deviations from the circular orbit (Fig. 11) have roughly the phase dependence of Figure 6 , with an amplitude $\sim 4 \mathrm{~km} \mathrm{~s}^{-1}$ peak-to-peak.

Now, we might ask, did Miller et al. really have data for TT Hya precise enough to detect the spurious eccentricity predicted for rotational-tidal distortion? I have tried fitting the velocities in their Table 2 and get comparable fits for circular and elliptical orbits $\left(\sigma \approx 6 \mathrm{~km} \mathrm{~s}^{-1}\right)$. If we restrict ourselves to their more precise data, those for 1994-2001, we can recover the elements listed in their Table 4 (col. [2]) to within the probable errors. However, this is only if we apply an unacknowledged $9.9 \mathrm{~km} \mathrm{~s}^{-1}$ zero-point "correction" to Peters' data. For a circular orbit fitted to this "corrected" data set, the deviations have roughly the phase dependence of Figure 6, but with the $2 \mathrm{~km} \mathrm{~s}^{-1}$ scatter superimposed.

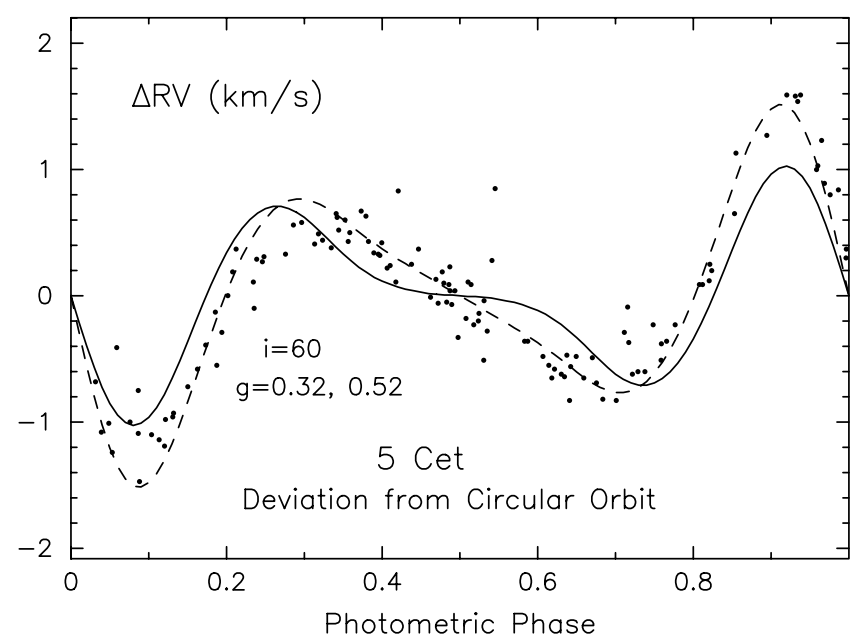

FIG. 10.-Deviation of measured radial velocities of $5 \mathrm{Cet}$ from a sine curve. Compare the shape of this curve to the right panel in Fig. 6. Dots are the measured deviations, the differences between the observations and fitted curve in Fig. 9. Curves give theoretically calculated deviations for two values of the gravity exponent, solid for $g=0.32$, dashed for $g=0.52$. Both calculations assume the mass ratio, 0.7 , derived from line broadening. 


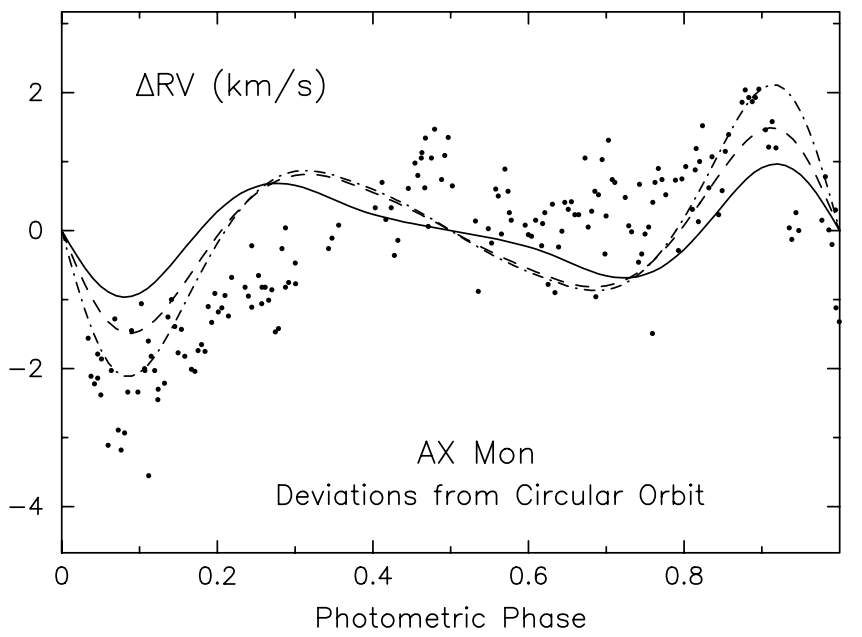

FIG. 11.-Deviation of measured radial velocities of AX Mon from a sine curve. Compare the shape of this curve to Fig. 6. As in Fig. 10, dots are the differences between observed velocities and a fitted sine curve. The curves give theoretically expected variations for $g=0.32$ with $i=60^{\circ}$ (solid), $g=0.52$ with $i=60^{\circ}$ (dashed), and $g=0.52$ with $i=75^{\circ}$ (dot-dashed). All assume $q=3.0$ and $x=0.77$.

The amplitude expected (for $K_{1}+K_{2}=167 \mathrm{~km} \mathrm{~s}^{-1}, i \sim 83$, $q=4.38, x=0.77$, and $g=0.32$ per Miller et al. [2007] and Van Hamme \& Wilson [1993]) is $\sim 8.2 \mathrm{~km} \mathrm{~s}^{-1}$ peak-to-peak, a bit smaller than the noisy observed value $\left(\sim 11 \mathrm{~km} \mathrm{~s}^{-1}\right)$.

\subsection{Some Further Applications}

We may use the calculations in $\S 3.1$ to restrict some of the parameters of Algol systems, given precise data. The amplitude of the deviations of the velocity curve from a sinusoid depends primarily on the orbital inclination, $i$, the velocity amplitude of the companion star, $K_{2}$, and the amount of gravity darkening, $g$. It also depends on limb darkening, but effectively much less than on $i$ or $g$, if we can assume the limb-darkening law is known as precisely as usually assumed in light-curve analyses Figure 6 shows that the deviations centered on superior conjunction (phases $0.75-0.25$ ) change much more with inclination than those centered on inferior conjunction (phases $0.25-0.75$ ). That dependence provides a way of restricting inclinations of the noneclipsing systems, such as 5 Cet and AX Mon, if we know $g$. In the eclipsing systems, on the other hand, we would get a moderately weak constraint on the mass ratio through $K_{2}$, although in these systems $q$ is usually very well constrained by the light curve. Wilson $(1979, \S$ VI) would argue that these constraints are built implicitly into his light-curve solutions. However, by stripping them out, we can isolate them and look at just what they might tell

TABLE 2

Some Measured Line Widths for 5 Cet

\begin{tabular}{|c|c|c|}
\hline Orbital Phase & $\begin{array}{c}\sigma_{\text {meas }} \\
\left(\mathrm{km} \mathrm{s}^{-1}\right)\end{array}$ & $\begin{array}{c}\sigma_{\text {calc }} \\
\left(\mathrm{km} \mathrm{s}^{-1}\right)\end{array}$ \\
\hline 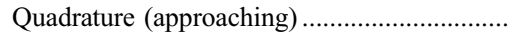 & 21.73 & 19.72 \\
\hline Quadrature (receding) & 21.71 & 19.87 \\
\hline Superior conjunction (star behind) .................... & 20.33 & 18.98 \\
\hline Inferior conjunction (star in front) ..................... & 20.03 & 18.51 \\
\hline
\end{tabular}

Note- The $\sigma$ s are the width parameter from a Gaussian fit to the line profile. The first value given for each phase comes from profiles in composite spectra of $5 \mathrm{Cet}$; the second value, from theoretically calculated profiles.

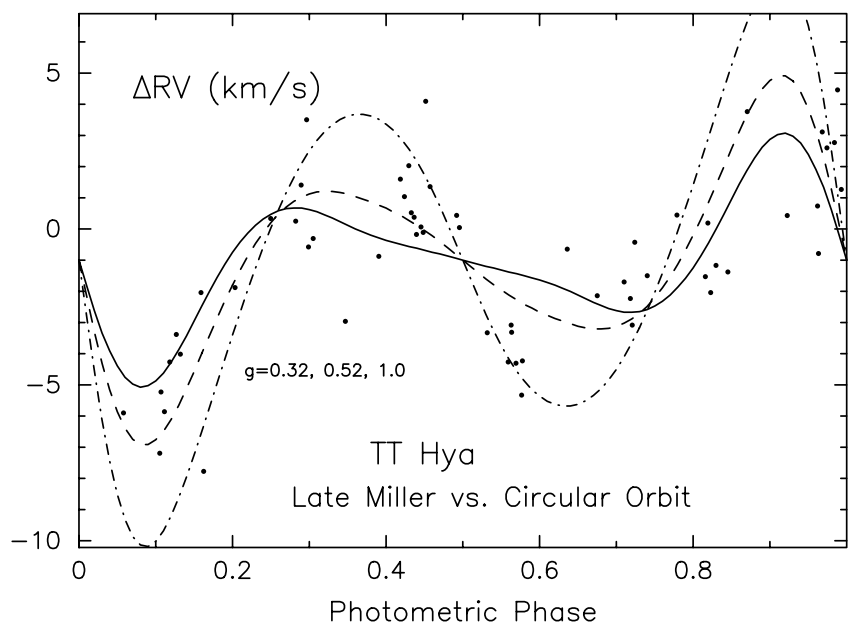

FIG. 12.-Deviation of measured radial velocities of TT Hya (the selected data from Miller et al. [2007] identified in § 3.2) from a sine curve. As in Fig. 10, dots are the differences between observed velocities and a fitted sine curve. The curves give theoretically expected variations for $g=0.32$ (solid), $g=0.52$ (dashed), and $g=1.0$ (dot-dashed).

us about various individual properties of a star and the physical processes that apply.

5 Cet gives us a good example of applying these velocitycurve distortions. In this system $\left(P=96.4\right.$ days, $K_{1}=24 \mathrm{~km} \mathrm{~s}^{-1}$, and $q \leq 0.82$, whence $M_{1} \sin ^{3} i \geq 1.50 M_{\odot}$ ) the inclination is probably fairly small to get reasonable masses. We previously estimated $i \sim 60^{\circ}$ (Eaton \& Barden 1988). For these present best guesses, the distortions predicted are somewhat smaller than observed (solid curve in Fig. 10). For AX Mon, we may get estimates of the properties of the system from Elias et al. (1997), namely $K_{1}+K_{2}=66-70 \mathrm{~km} \mathrm{~s}^{-1}, i=50^{\circ}-70^{\circ}$, and $q=4.0-$ 3.0. For values characterizing this range $\left(i=60^{\circ}\right.$ and $q=3.0$, whence $K_{1}+K_{2}=69 \mathrm{~km} \mathrm{~s}^{-1}$ ), the calculated curve for $g=$ 0.32 is much too shallow to fit the observations. Even if we increase $g$ to 0.5 , the inclination is too small. Instead, the inclination is probably larger, or the gravity darkening even larger than it seems for 5 Cet. Thus the velocity-curve distortions constrain the properties of this noneclipsing binary to the ends of the ranges expected from photometry, namely to $q \approx 3.0$ and $i \approx 75^{\circ}$.

The rather strong differences of the curves in Figure 8 gives us what is perhaps a unique test of Lucy's (1967) theory of gravity darkening for convective stars. This brilliantly simple theory predicts $g=0.32$ for convective stars; more detailed calculations by Claret $(2000,2003)$ for a wide range of convective stars predict essentially the same level of gravity darkening or slightly less. A simple comparison of the observed velocity-curve distortions in Figures 10-12 with the theoretical curves in Figure 8 shows the binary components must be gravity darkened. On the other hand, the relative amplitudes of the effect for the two conjunctions in the observations is much lower than for radiative gravity darkening $(g=1.0)$. The amount of gravity darkening is hard to measure because of the residual scatter in the data and because two of our three stars have indeterminate inclinations. However, in Figure 10 the shape of the observed curve is much closer to the shape calculated for $g=0.52$ than for 0.32. In Figure 11, as we have seen, a similar increase in $g$ gives the observed amplitude without requiring $i$ to be unacceptably large. In Figure 12 , for a system in which the geometry is very highly constrained, $g=0.52$ fits the amplitude much better than $g=0.32$ and $g=1.0$ does not fit it at all. So, the amount of gravity darkening seems to be around $g=0.5$, a little larger than Lucy's 
$g=0.32$. It should be possible to constrain the gravity darkening of convective stars much better in future with precise radial velocities for more eclipsing stars.

Tidal distortion should give a variable $v \sin i$ for a binary component because the disc is actually broader at some phases than at others. We saw this effect in UU Cnc (Eaton et al. 1991). The new data for 5 Cet provide a further test, and I have applied it by looking at composite spectra of 5 Cet at the two conjunctions and at the two quadratures. First, blends of Fe lines near 6575 and $6594 \AA$, which can give a very good measure of $v \sin i$ without numerically measuring and calibrating line widths (Eaton 1990), are somewhat sharper at the conjunctions than at the two quadratures. Second, widths of Gaussians fit to cross-correlation functions (of a list of strong solar absorption lines with the observed spectrum; Eaton \& Williamson 2007) for these four composite spectra are about $5 \%$ broader for the quadratures than the conjunctions. See Table 2 for the details. We may simulate this effect by calculating synthetic spectra and measuring the variation of line width with phase, and I have done so for a rough model for the system. This model assumes the visible giant star is a contact component with $x=0.70$ and $g=0.32$, and that $K_{1}=$ $23.9 \mathrm{~km} \mathrm{~s}^{-1}$ and $q=0.8$. I used a spectrum of $\delta$ Eri (K0 IV) from the National Solar Observatory covering the wavelength range $6400-6480 \AA$ to represent the cool component in this calculation, but the resolution is similar to that in the AST observations. The calculated line widths (col. [3] of Table 2) have roughly the same phase dependence as shown by the observations but are about $10 \%$ narrower. We can make them as large as the observed widths by reducing the mass ratio to about $q=$ 0.7 , thereby raising $K_{1}+K_{2}$. In any case, a mass ratio greater than 1.0 (unseen star more massive than the contact component) gives profiles much narrower than observed.

\section{SUMMARY AND DISCUSSION}

The existence of precise radial velocities for long-period binaries, made possible by a dedicated instrument on a robotic telescope, has made it possible to demonstrate the distortion of the radial-velocity curves of close binaries predicted by Sterne (1941). The distortions depend on a binary's inclination and mass ratio in ways that let us estimate these properties in favorable circumstances. Given the nature of this velocity-curve distortion, we must conclude that there is no credible evidence for orbital eccentricity in binaries with contact components. Furthermore, the distortion observed in three systems requires the stars to be gravity darkened, probably somewhat more than expected from Lucy's (1967) theory but much less than expected for stars with radiative envelopes.

In addition to the foregoing substantive results, I have also (1) detected the variation of $v \sin i$ caused by tidal distortion in 5 Cet and used it to derive an improved mass ratio, $M_{\text {unseen }} / M_{\mathrm{gK}} \approx 0.70,(2)$ estimated somewhat improved geometrical properties for AX Mon, and (3) explored what properties a binary encased in a common envelope would have if we ever were to detect one.

\subsection{A Caveat about Limb Darkening}

The greatest uncertainty in the results of this paper, as well in many others of the same genre, comes from the effect of limb darkening. This uncertainty would impact both papers analyzing light curves and those based on rotational line broadening or Doppler images. Such analyses usually parameterize the emergent intensity as a function of the angle between the surface normal and the line of sight, more specifically on $\mu$, its cosine.
The simplest formulation, a linear dependence on $\mu$, follows from the stratification of a simple hydrostatic atmosphere in radiative equilibrium. Other more complicated formulations are possible with increasingly less intuitive mapping into the atmospheric structure. Eventually, these effectively seem to become mere fitting schemes (e.g., Brown et al. 2001). Alternatively, one may apply a calculated intensity directly, perhaps by looking it up in a table of $I_{\lambda}$ vs. $\mu$.

Theoretical model atmospheres predict levels of limb darkening that may be applied in analyses of binary stars and other problems. Such analyses usually assume limb-darkening coefficients from a few standard theoretical lists (e.g., Al-Naimy 1978; Claret \& Gimanez 1990). Normally, such models assume planeparallel stratification in hydrostatic equilibrium, basically the physics we apply to the Sun. A good example is the grid of atmospheres Kurucz calculated with his program ATLAS (e.g., Kurucz 1995). A recent alternative set of models is NextGen (Hauschildt et al. 1999a, 1999b). This latter grid ought to give a superior interpretation of our standard physical assumption; for example, by calculating line blanketing in the blue-UV through opacity sampling. Limb darkening from NextGen, especially from the spherical models for giants and supergiants (Claret \& Hauschildt 2003), is quite different than from plane-parallel models. It tends to be much more pronounced, with the intensity dropping to zero well before the limb (see Orosz \& Hauschildt 2000, their Fig. 4).

If real stars really have the limb darkening of these spherical NextGen models, there would be systematic errors in a wide range of observational results for giants and supergiants. For instance, it would make direct measurements of stellar diameters systematically small, leading to effective temperatures that are too high. This biases the Barnes-Evans relation (Barnes \& Evans 1976), used extensively to derive angular diameters for cool stars and otherwise calibrate the flux-temperature relationship. This effect might be tested by analyzing the consistency of temperatures derived by fitting spectral energy distributions (e.g., Bertone et al. 2004) with those derived from measured bolometric fluxes and angular diameters. It would likewise bias rotational velocities from line broadening and a wide range of properties of binaries derived from such broadening such as mass ratios of noneclipsing systems, as discussed by Orosz \& Hauschildt (2000) and Shahbaz (2003). It would also bias the results for eclipsing systems by making it harder to fit the eclipse shapes and ellipsoidal variation simultaneously. Furthermore, it might well bias the Doppler images of spotted stars, if the effects are as extreme as calculations of Claret \& Hauschildt (2003, see their Fig. 2) imply. In our present analysis, the effect of the nontraditional limb darkening of NextGen models would likely be to increase the amplitude of the velocity variation somewhat, allowing for a lower gravity darkening. We can see this effect by making the linear limb-darkening coefficient $x>1.0$ and truncating the intensity at zero when it becomes negative near the limb. Such a calculation does increase the deviation from a sinusoidal velocity curve.

Now then, how much do we have to worry about the existing body of lore for stellar astronomy? Perhaps not as much as one would fear. There are few highly precise determinations of limb darkening for cool stars other than the Sun, and that is especially true for giants and supergiants. However, one reliable determination for a $\mathrm{K}$ giant (Fields et al. 2003) finds limb darkening much less extreme than predicted by NextGen models. Predictions of ATLAS were somewhat closer, but did not fit as well as one might hope. Fields et al. suspected unmodeled physical effects are to blame for this discrepancy, inasmuch as there are obvious effects in atmospheres beyond our current understanding. None of these models, for instance, incorporate the inhomogeneities 
that we expect in all stellar atmospheres on the basis of solar granulation and Ayres's (e.g., 2002) work on CO. Furthermore, all these calculations necessarily use very simplistic models for turbulence, whatever that really is, and for convection. At this point, it seems the theoretical limb darkening of NextGen models is not tested well enough for us to worry seriously about its effect on binary-star analyses, but that we as a community should take it seriously enough to look for more tests, especially for the supergiants.
I would like to thank Peter Wood for stimulating me to think about this problem, for giving me a key reference to work on the long-period variations of AGB stars, and for clarifying some details of his work on rotating spheriods. This research used the SIMBAD database and was supported by NASA grants NCCW0085 and NCC5-511 and by NSF grants HRD 9550561 and HRD 9706268.

\section{Facilities: TSU:AST}

Al-Naimy, H. M. 1978, Ap\&SS, 53, 181

Ayres, T. R. 2002, ApJ, 575, 1104

Barnes, T. G., \& Evans, D. S. 1976, MNRAS, 174, 489

Bertone, E., Buzzoni, A., Chávez, M., \& Rodriguez-Merino, L. H. 2004, AJ, 128,829

Brown, T. M., Charbonneau, D., Gilliland, R. L., Noyes, R. W., \& Burrows, A. 2001, ApJ, 552, 699

Claret, A. 2000, A\&A, 359, 289 2003, A\&A, 406, 623

Claret, A., \& Gimanez, A. 1990, A\&A, 230, 412

Claret, A., \& Hauschildt, P. H. 2003, A\&A, 412, 241

Derekas, A., Kiss, L. L., Bedding, T. R., Kjeldsen, H., Lah, P., \& Szabo, Gy. M. 2006, ApJ, 650, L55

Eaton, J. A. 1990, Inf. Bull. Var. Stars, 3460, 1

Eaton, J. A., \& Barden, S. C. 1988, Acta Astron., 38, 353

Eaton, J. A., Hall, D. S., \& Honeycutt, R. K. 1991, ApJ, 376, 289

Eaton, J. A., Henry, G. W., Bell, C., \& Okorogu, A. 1993, AJ, 106, 1181

Eaton, J. A., \& Williamson, M. H. 2007, PASP, 119, 886

Elias, N. M., Wilson, R. E., Olson, E. C., Aufdenberg, J. P., Guinan, E. F., Guedel, M., van Hamme, W. V., Stevens, H. L. 1997, ApJ, 484, 394

Fields, D. L. et al. 2003, ApJ, 596, 1305

Hall, D. S. 1990, AJ, 100, 554

\section{REFERENCES}

Hauschildt, P. H., Allard, F., \& Baron, E. 1999a, ApJ, 512, 377

Hauschildt, P. H., Allard, F., Ferguson, J., Baron, E., \& Alexander, D. R. 1999b, ApJ, 525, 871

Hinkle, K. H., Lebzelter, T., Joyce, R. R., \& Feckel, F. C. 2002, AJ, 123, 1002 Kopal, Z. 1959, Close Binary Systems (London: Chapman \& Hall)

Kurucz, R. L. 1995, Kurucz CD-ROM 13, ATLAS9 Stellar Atmosphere Programs and $2 \mathrm{~km} / \mathrm{s}$ Grid (Cambridge: SAO)

Lucy, L. B. 1967, Z. Astropys., 65, 89

Lucy, L. B., \& Sweeney, M. A. 1971, AJ, 76, 544 1973, Observatory, 93, 37

Miller, B., Budaj, J., Richards, M., Koubský, P., \& Peters, G. J. 2007, ApJ, 656, 1075

Olivier, E. A., \& Wood, P. R. 2003, ApJ, 584, 1035

Orosz, J. A., \& Hauschildt, P. H. 2000, A\&A, 364, 265

Russell, H. N. 1912, ApJ, 36, 54

Shahbaz, T. 2003, MNRAS, 339, 1031

Sterne, T. C. 1941, Proc. Natl. Acad. Sci., 27, 168

Van Hamme, W., \& Wilson, R. E. 1993, MNRAS, 262, 220

Wilson, R. E. 1979, ApJ, 234, 1054

Wilson, R. E., \& Sofia, S. 1976, ApJ, 203, 182

Winn, J. N., et al. 2005, ApJ, 631, 1215

Wood, P. R., Olivier, E. A., \& Kawaler, S. D. 2004, ApJ, 604, 800 